\title{
Spectroscopic properties of post-AGB stars
}

\author{
M. Parthasarathy \\ Indian Institute of Astrophysics, Bangalore - 560034, India; \\ Department of Physics and Astronomy, University of Oklahoma, Norman, OK 73019, USA \\ email: partha@iiap.res.in
}

Abstract. A review of the spectroscopic properties of post-AGB stars is presented.

Keywords. stars : post-AGB, evolution, abundances, binaries, mass-loss, circumstellar-matter

\section{Introduction}

Post-AGB stars (proto-planetary nebulae; PPNe) are objects that have recently evolved off the AGB (after the termination of the AGB phase of evolution of low and intermediate mass stars) but have not yet reached high enough temperatures to photoionize their circumstellar envelopes. This evolutionary stage is very short-lived, depending on the core mass ( Schönberner 1983, Blöcker 1995). The low and intermediate mass stars evolve through the post-AGB phase and planetary nebulae (PNe) phase and end up as white dwarfs. During the transition from the tip of the AGB to PN phase they appear as M-, K-, G-, F-, A- and OB-type post-AGB supergiants for a short period (Parthasarathy and Pottasch 1986, 1989; Parthasarathy 1993a,b; Kwok 1993; Van Winckel 2003).

\section{Very metal-poor post-AGB A-supergiants}

The photospheric abundances of very metal-poor post-AGB A-supergiants show that the refractory elements $\mathrm{Fe}, \mathrm{Mg}, \mathrm{Si}, \mathrm{Al}, \mathrm{Ti}, \mathrm{Ca}$, etc., are depleted and the abundances of volatile elements $\mathrm{C}, \mathrm{N}, \mathrm{O}, \mathrm{S}$, and $\mathrm{Zn}$ do not show such extreme depletions. The photospheric abundances of these stars correlate with the grain condensation temperatures (Bond 1991; Van Winckel et al. 1992; Parthasarathy et al. 1992; Waelkens et al. 1996). The Zn abundances in the extremely Fe-poor post-AGB stars are (see Takeda et al. 2002 and references therein): HD $52961([\mathrm{Zn} / \mathrm{H}]=-1.5,[\mathrm{Fe} / \mathrm{H}]=-4.8), \mathrm{HR} 4049([\mathrm{Zn} / \mathrm{H}]$ $=-1.3,[\mathrm{Fe} / \mathrm{H}]=-4.7)$, and $\mathrm{HD} 44179([\mathrm{Zn} / \mathrm{H}]=-0.6,[\mathrm{Fe} / \mathrm{H}]=-3.3) . \mathrm{S}$ and $\mathrm{Zn}$ have very low condensation temperatures and therefore do not easily condense into dust grains. From Figure 6 and Table 2 of Takeda et al. (2002) it is clear that the abundance of Zn traces the original metallicity of stars that show the depletion of refractory elements.

Cami and Yamamura (2001) discovered anomalous oxygen isotopic ratios in HR 4049. They find ${ }^{16} \mathrm{O} /{ }^{17} \mathrm{O}=8.3$ and ${ }^{16} \mathrm{O} /{ }^{18} \mathrm{O}=6.9$. These values are at least one order of magnitude lower than any previously determined isotopic ratio in any type of evolved star. These anomalous oxygen isotopic ratios in HR 4049 cannot be explained with current nucleosynthesis models. Recent studies of several RV Tauri stars (Maas et al. 2005; De Ruyter et al. 2006; Giridhar et al. 2005) show that the depletion of refractory elements in their photospheres is linked to the presence of binary companions and circumbinary dust disks (De Ruyter et al. 2006; Jura 2005). The above-mentioned abundance studies of RV Tauri stars reveal that there is no detectable overabundance of s-process elements. This result indicates that RV Tauri stars are not evolved from luminous AGB phase objects. Their CNO abundances are close to that expected from giants after the first 
dredge-up. Deroo et al. (2005) find that V $453 \mathrm{Oph}$ is an s-process enriched ([s/Fe] = $+0.5)$ carbon-poor RV Tauri star of low intrinsic metallicity $([\mathrm{Fe} / \mathrm{H}]=-2.2)$. The low $\mathrm{N}$ abundance excludes $\mathrm{CN}$ cycling as being the cause of the low $\mathrm{C}$ abundance. It may not be a typical RV Tauri type star. It may be a carbon-poor first ascent giant in a close binary system with mass transfer from an evolved companion.

\subsection{Binarity of very metal-poor A-F type post-AGB stars}

There is conclusive evidence that all the known very metal-poor post-AGB A- and Fsupergiants (HR 4049, HD 52961, HD 44179, etc.) and RV Tauri stars with depleted refractory elements in their photospheric abundances are single-lined spectroscopic binaries with orbital periods of several hundred days (Van Winckel 2003). In the case of HR 4049, Monier and Parthasarathy (1999) found that its UV energy distribution is very deficient compared to that of a standard A6Ib star or an ATLAS 9 model $\left(\mathrm{T}_{\text {eff }}=\right.$ $7500 \mathrm{~K}, \log \mathrm{g}=2.0$ and $[\mathrm{Fe} / \mathrm{H}]=-5.0)$ which best fits the optical continuum. The UV-flux deficiency of HR 4049 scales as $\lambda^{-1}$ (Monier and Parthasarathy 1999; Waelkens et al. 1995). The UV and optical light variations of HR 4049 are found to occur in phase as a result of variable circumstellar extinction (Monier and Parthasarathy 1999; Waelkens et al. 1995). The high galactic latitude $\left(\mathrm{b}=57^{\circ}\right)$ post-AGB star HD 213985 (A2Ia) also shows variable optical and UV circumstellar extinction (Waelkens et al. 1995). These variations are related to the circumbinary dust disks in these systems. However there are several A- and F-type post-AGB stars with dust disks (Sivarani 2000) and they do not show depletion of refractory elements in their chemical composition. For example HD 101584, which is a F0Ia post-AGB supergiant (Sivarani et al. 1999) with hot and cold circumstellar dust disk and a bipolar outflow, does not show depletion of refractory elements in its photospheric spectrum.

The A-type post-AGB binary HD 44179 (Cohen et al. 2004) is associated with a bipolar proto-planetary nebula of biconical appearance known as the Red Rectangle. It is a single-lined spectroscopic binary with an orbital period of 319 days (Van Winckel 2003). The high resolution HST images reveal a dusty disk and complex structures (Cohen et al. 2004), many of them unique to this object. The Red Rectangle and the central A-type post-AGB star are unique spectroscopically. Its spectrum shows many unidentified optical, molecular bands in emission (Van Winckel et al. 2002), superposed on a continuous Extended Red Emission (ERE). The carrier of the ERE is still under investigation (Witt and Vijh 2004). Schultheis et al. (2002) found two or three A and B stars with mid-IR excesses whose optical spectra indicate the presence of ERE. Further study of these stars is needed. These stars may be similar to HD 44179.

HD 172481 (Reyniers and Van Winckel 2001), Hen 3-1312 (Pereira 2004), IRAS 085444431 (Maas et al. 2003) and IRAS 10215-5916 (AFGL 4106) (Molster et al. 1999; Sivarani 2000) were found to be F-type post-AGB binaries with circumstellar dust envelopes. Analysis of high resolution spectra of the F-type post-AGB candidates IRAS 19386+0155 (Pereira et al. 2004) and IRAS 19475+3119 (HD 331319) (Klochkova et al. 2002, Sivarani 2000) reveals abundance peculiarities in IRAS $19386+0155$ and the abundances in HD 331319 appear to be similar to those of HD 161796. Infrared data on these two stars indicates the presence of dust disks. HD 179821 (IRAS 19114+0002) (Pottasch and Parthasarathy 1988; Reddy and Hrivnak 1999; Thevenin et al. 2000; Jura et al. 2001) and HD 168625 (IRAS 18184-1623) (Sivarani 2000) are classified as post-AGB supergiants and also as massive Pop. I supergiants. Further study of these stars is needed to understand their evolutionary status (cf. poster paper by Bobrowsky et al.). 


\section{F-type post-AGB stars with overabundances of carbon and s-process elements}

Post-AGB stars which have gone through the carbon-star stage on the AGB are expected to show the products of the triple-alpha process, $\mathrm{CN}$ and $\mathrm{ON}$ cycling and the s-process on the surface, as a result of thermally pulsing AGB evolution and the third dredge-up. Detailed abundance analyses have been made for 12 carbon and s-process rich post-AGB stars (Reyniers et al. 2004; Reddy et al. 2002). F-type post-AGB stars with 21- $\mu \mathrm{m}$ emission features in the IR are all overabundant in carbon and s-process elements (Parthasarathy et al. 1992; Van Winckel 2003; Reddy et al. 1997, 2002; Reyniers et al. 2004). This group of post-AGB stars shows a clear relation between neutron irradiation and the s-process overabundances, based on the ratio of Barium peak elements (heavy s-process elements) to Sr-peak elements (light s-process elements). From a comparison with the predictions of AGB models, Reyniers et al. (2004) suggest that the observed spread in nucleosynthesis efficiency is intrinsic and indicates that different ${ }^{13} \mathrm{C}$ pockets are needed for stars with comparable mass and metallicity to explain their abundances. The large spread in the s-process abundances of post-AGB stars having similar parameters is not understood. New models of nucleosynthesis and mixing in AGB stars are needed. Parthasarathy, Reddy, Garcia-Lario (in preparation) analysed the high resolution spectrum of the post-AGB F-supergiant in CRL 2688. They find $\mathrm{T}_{\text {eff }}=7000 \mathrm{~K}$, $\log \mathrm{g}=0.5,[\mathrm{Fe} / \mathrm{H}]=-0.33$. The total $\mathrm{CNO}=9.16$ and $\mathrm{C} / \mathrm{O}=1.6$ clearly indicates that CRL 2688 has gone through the third dredge-up and carbon star stage on the AGB. There is no depletion of refractory elements as indicated by the abundance of $\mathrm{Zn}$ $[\mathrm{Zn} / \mathrm{H}]=-0.48$. The s-process elements Ba, Y, Zr, etc., are significantly overabundant. Reyniers et al. (2002), from an analysis of VLT UVES spectra, find that the line at $6708.1 \AA$ seen in the s-process enriched post-AGB stars described above is in fact due to the Ce II line at $6708.099 \AA$, and not to Li I. It indicates the importance of complete heavy element line lists in the analysis of spectra of strongly s-process enriched post-AGB stars. The above mentioned study clearly shows that for the s-process enriched post-AGB stars there is no Li line in their spectra and no Li production.

There are several post-AGB stars which are found to show no overabundance of sprocess elements. Their photospheres and circumstellar dust shells are found to be oxygen rich indicating the absence of efficient third dredge-up in these stars. All the post-AGB stars with $21-\mu \mathrm{m}$ emission features are found to show high $\mathrm{C} / \mathrm{O}$ ratios and overabundances of s-process elements. These stars have evolved through the carbon star stage on the AGB and they may evolve into [WC] type PNe (Parthasarathy 1999). Some of the most s-process enriched F-type post-AGB stars are : IRAS $05341+0852([\mathrm{Fe} / \mathrm{H}]=-0.7$, $([\mathrm{s}-$ process $/ \mathrm{Fe}]=+2.2)$ (Reddy et al. 1997), and IRAS 06530-0213 $([\mathrm{Fe} / \mathrm{H}]=-0.5$, $[$ s-process $/ \mathrm{Fe}]=+2.06$ ) (Reyniers et al. 2004). The optical spectra of these two stars are completely dominated by atomic transitions of s-process elements. For low-metallicity stars $([\mathrm{Fe} / \mathrm{H}]<-1)$ the s-process nucleosynthesis can lead to large overabundances of lead $(\mathrm{Pb})$ with respect to other s-process elements such as Barium (Ba). Low-metallicity $([\mathrm{Fe} / \mathrm{H}]<-1) \mathrm{A}$ and $\mathrm{F}$ type post-AGB stars with overabundances of carbon and sprocess elements are expected to show overabundance of lead $(\mathrm{Pb})$. The discovery and study of such stars will enable us to further understand s-process nucleosynthesis.

\section{Hot post-AGB stars}

The G-, F- and A-type post-AGB stars evolve towards the left in the HR-diagram at constant luminosity into hotter post-AGB stars. As a result of photoionisation of the 
surrounding circumstellar dust and gas shell by the hot post-AGB star, they eventually appear as planetary nebulae with hot post-AGB central stars. From the analysis of IRAS data and spectra of IRAS sources with far-IR colours similar to post-AGB stars and PNe, several new hot post-AGB stars have been identified (Parthasarathy et al. 2000a; Parthasarathy et al. 2001b; Parthasarathy and Pottasch 1989; Parthasarathy 1993a,b). Gauba \& Parthasarathy (2003) studied the UV(IUE) spectra of several high galactic latitude hot post-AGB stars. In many cases, the UV spectra suggested partial obscuration of the hot central stars due to circumstellar dust. The circumstellar extinction law in these cases was found to be linear in $\lambda^{-1}$ suggesting an upper limit of $\sim 200 \AA$ for the radii of the small grains. Carbon-rich and oxygen-rich features were identified in the ISO spectra of these objects (Gauba \& Parthasarathy 2004). Klochkova et al. (2002) find IRAS $01005+7910$ to be a high galactic latitude hot post-AGB star with an overabundance of carbon. Sarkar et al. (2005) analysed the high resolution spectra of the hot post-AGB candidates IRAS 13266-5551 (CPD -55 5588) and IRAS 17311-4924 (Hen3-1428). The above mentioned three stars show evidence for post-AGB mass-loss and for the presence of low excitation nebulae.

Sarkar, Parthasarathy \& Garcia-Lario (in preparation) analysed the high resolution spectrum of the hot post-AGB star IRAS22023+5249 (LS III +52 24). H I, He I, C II and Fe III lines were found to show P-Cygni profiles indicating post-AGB mass loss. Low excitation nebular lines are also present in the spectrum in addition to forbidden and permitted emission lines of Fe, etc. Absorption lines of O II, He I, Si III, N II and C III are used to derive atmospheric parameters $\left(\mathrm{T}_{\text {eff }}=24000 \mathrm{~K}, \log \mathrm{g}=3.0\right)$ and abundances. The CNO abundances are found to be more than those of main sequence B stars in Ori OB1. The IUE UV spectra of the bipolar PPNe IRAS 17423-1755 (Hen 3-1475) and IRAS 10178-5958 (Hen 3-401) indicate that the central B-type stars are obscured by dusty disks. The UV flux of these two stars in the wavelength interval $1150 \AA$ to $1900 \AA$ is very weak or absent (Gauba and Parthasarathy 2003; Parthasarathy et al. 2001a). The optical spectra of these two stars show several emission lines and low excitation nebular emission lines, indicating the presence of B-type central stars (Riera et al. 2003; GarciaLario et al. 1999). In the case of Hen 3-401, the UV spectrum shows a stellar continuum in the wavelength interval $2400 \AA$ to $3200 \AA$, which corresponds to a moderately reddened B8 type central star (Parthasarathy et al. 2001a). Sahai et al. (1999) from HST high resolution images find two very long bipolar outflow lobes. The central star and disk are also revealed. The optical spectra, HST imaging and spectroscopic data of Hen 31475 shows the presence of high velocity outflow and $1200 \mathrm{~km} \mathrm{~s}^{-1}$ jets (Borkowski and Harrington 2001; Riera et al. 2003).

Several hot post-AGB stars have also been identified at high galactic latitudes and in the halo of our Galaxy on the basis of their derived atmospheric parameters and chemical composition (Mooney et al. 2002 and references therein). However the abundance patterns of hot post-AGB stars found in the halo of our Galaxy show significant differences when compared with the abundance patterns of G- , F- and A-type post-AGB stars. Most significant is the underabundances of carbon in the halo hot post-AGB stars. Such carbon underabundances are also observed in the hot-post AGB stars in globular clusters. Thus it is likely that the hot post-AGB stars with underabundance of carbon have left the AGB before undergoing the third dredge-up.

\section{Rapidly evolving hot post-AGB stars}

The best examples of rapidly evolving A- and B-type post-AGB stars are: Henize $1357=$ IRAS 17119-5926 = SAO $244567=$ Stingray Nebula, and SAO $85766=$ IRAS 
$18062+2410$. Less than 100 years ago they were A-type post-AGB stars and now they are OB central stars of low excitation planetary nebulae.

\subsection{Henize 1357 = Stingray Nebula}

Hen 1357 was the first object that was shown to have evolved from a B1 type postAGB supergiant into a PN in the extremely short timescale of 20 years (Parthasarathy et al. 1993, 1995, Bobrowsky et al. 1998). The UV (IUE) spectra of Hen 1357 (Figure 1) shows the rapid evolution of the central star from 1988 to 1996. The P-Cygni profiles of N V $(1240 \AA)$ and C IV $(1550 \AA)$ in the spectra taken in 1988 had indicated a terminal wind velocity of $-3500 \mathrm{~km} \mathrm{~s}^{-1}$. The wind appears to have completely ceased by 1994 (Fiebelman 1995; Parthasarathy et al. 1995). We find that the object has faded by a factor of 3 in the UV, from 1988 to 1996. The fading suggests that the central star of Hen 1357 is rapidly evolving into a DA white dwarf (Parthasarathy et al. 1995). The luminosity, the core mass, observed rapid evolution and fading of of Hen 1357 are not in agreement with the timescales of evolution of low or high mass post-AGB models. Recent HST images of Hen 1357 (Bobrowsky et al. 1998) showed the presence of a $1.67^{\prime \prime} \times 0.92^{\prime \prime}$ nebula around the central star. The HST images also revealed the presence of collimated outflows and a binary companion to the central star of Hen 1357 . The object needs to be monitored in the UV, optical and IR during the next few decades in order to understand the birth and early evolution of PNe and their central stars.

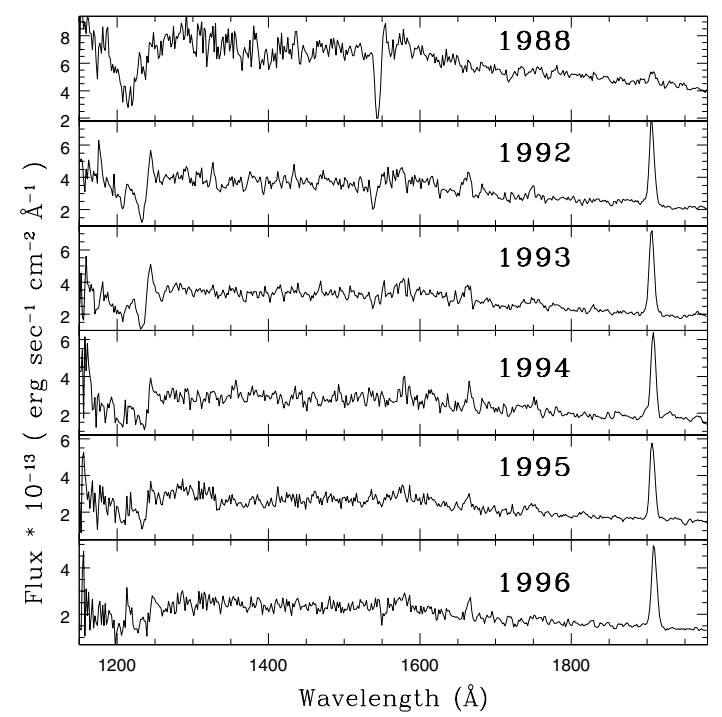

Figure 1. UV(IUE) spectra of Hen 1357. Notice the variation in the N v (1240 $\AA$ ), C IV

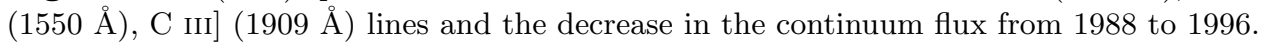

\section{2. $S A O 85766$}

SAO 85766 (=HDE $341617=$ V886 Her=IRAS 18062+2410; Volk and Kwok 1989) appears to have rapidly evolved in the last 75 years (Arkhipova et al. 1999; Parthasarathy et al. 2000b) similar to the case of Hen 1357 described above. In the HDE catalogue HDE $341617=$ SAO 85766 was classified as an A5 star, based on the plate taken in July 1924 (JD 2423971) (Arkhipova et al. 1999). Now it is a B1 supergiant with a low excitation nebula (Arkhipova et al. 1999, 2001a; Parthasarathy et al. 2000b). Its A5 spectral type in 1924 indicates that its $\mathrm{T}_{\text {eff }}$ in 1924 was $8500 \mathrm{~K}$; now the $\mathrm{T}_{\text {eff }}$ based on its B1I type 
spectrum and the analysis of recent high resolution spectra yields $\mathrm{T}_{\text {eff }}=22000 \mathrm{~K}$. The significant underabundance of carbon indicates that SAO 85766 left the AGB before undergoing a third dredge-up (Parthasarathy et al. 2000b, Mooney et al. 2002; Ryans et al. 2003). SAO 85766 shows irregular rapid light variations of low amplitude due to pulsation (Arkhipova et al. 1999, 2001a) (cf. Poster paper by Arkhipova et al.). In the UV also similar light variations are detected (Gauba and Parthasarathy 2003).

\section{3. $L S I I+3426$ and $L S I V-12111$}

LS II + 3426 (IRAS20462+3416 = V1853 Cyg) was classified as a massive B1.5 Ia - Iabe supergiant by Turner and Drilling (1984). They estimated its distance to be $17.8 \mathrm{kpc}$ by assuming that it is a massive population I B supergiant located in the outer edge of the Galaxy. Parthasarathy (1993a) discovered it to be an IRAS source with far-IR (IRAS) colours similar to A- and F-type post-AGB supergiants and PNe. Based on its far-IR colors, global energy distribution, low gravity and high galactic latitude, Parthasarathy (1993a) suggested that LS II +34 26 (IRAS20462+3416) is a low-mass post-AGB B-type supergiant surrounded by a cold detached dust envelope, rather than a massive population I B supergiant. Parthasarathy (1993a) suggested that the B-supergiant spectrum of LS II + 3426 shows an evolutionary sequence of post-AGB supergiants from cooler $(\mathrm{G}, \mathrm{F}$, A) to hotter (B and O) types. The nebular emission lines in the optical spectrum of LS II +3426 indicated that it is rapidly evolving into a very low excitation PN (Parthasarathy 1994, Garcia-Lario et al. 1997). Such nebular emission lines seem to be absent in the spectrum of LS II + 3426 obtained by Turner and Drilling (1984) in 1977. Differences in the spectrograms obtained by Turner and Drilling in 1977 and 1981 and the recent spectra of LS II +34 26 (Parthasarathy 1994; Garcia-Lario et al. 1997; Arkhipova et al. 2001b) suggest that it is rapidly evolving into the PN stage. A multiwavelength study of the object (García-Lario et al. 1997) confirmed that the central star is surrounded by a very low excitation and compact nebula (Ueta et al. 2000). The presence of P-Cygni profiles indicates ongoing post-AGB mass-loss (Garcia-Lario et al. 1997 and Arkhipova et al. 2001b). Like Hen 1357 and SAO 85766, LS II +34 26 seems to be another case of a rapidly evolving hot post-AGB star. Recently Arkhipova et al. (2001b) made photometric and spectroscopic observations of LSII +34 26 and found rapid irregular low amplitude light variations similar to that of SAO 85766 (Arkhipova et al. 1999, 2001a; and poster paper). The spectrum, chemical composition and evolutionary stage of LS IV -12 111 (IRAS 19590-1249; Ryans et al. 2003) is very similar to that of SAO 85766 and LS II +34 26, showing a significant underabundance of carbon. Similar underabundances of carbon have been reported in other hot post-AGB stars at high galactic latitudes. These stars left the AGB before the third dredge-up.

\section{Post-AGB stars in globular clusters}

Recent studies indicate that some of the UV-bright stars (Zinn et al. 1972; Harris et al. 1983; de Boer 1987) in globular clusters are in a post-AGB stage of evolution (Ambika et al. 2004; Jasniewicz et al. 2004; Mooney et al. 2004). Chemical composition studies of B-, A- and F-type UV-bright stars in globular clusters reveal that some of them are postAGB A and F stars showing evidence for third dredge-up and overabundances of carbon and s-process elements, for example ROA 24 in Omega Cen (Gonzalez and Wallerstein 1994) and PN K648 in M15 (Bianchi et al. 2001), while some hot post-AGB stars show severe carbon deficiencies, indicating that they left the AGB before the third dredge-up occurred, for example ZNG 1 in M10, ROA 5701 in Omega Cen and Barnard 29 in M13 (Moehler et al. 1998; Jasniewicz et al. 2004; Mooney et al. 2004, Thompson et al. 2006). 


\section{Post-AGB binary stars and Type Ia supernovae}

SN 2002ic (Hamuy et al. 2003, Wood-Vasey et al. 2004) is the first Type Ia supernova whose spectrum displayed strong circumstellar hydrogen lines. Wang et al. (2004), WoodVasey et al. (2004) and Kotak et al. (2004) suggested that the SN 2002ic progenitor was a post-AGB binary system. Observations indicate that the SN 2002ic event took place inside a dense clumpy, aspherical disk-like circumstellar environment. All these characteristics are present in some of the post-AGB binary systems with dusty disks. The exact progenitors of Type Ia SNe have not been identified (Branch et al. 1995). Single degenerate scenario is generally favoured today and some of the post-AGB binary systems can be candidate progenitors of Type Ia SNe similar to SN 2002ic. Detailed studies of a large sample of cool and hot post-AGB stars may reveal a few post-AGB binary systems which can be considered as candidate progenitors for Type Ia SNe similar to SN 2002ic.

\section{Acknowledgements}

I am very thankful to Prof. David Branch, Prof. Ryan Doezema and Prof. E. Baron for their kind support, encouragement and hospitality.

\section{References}

Ambika S., Parthasarathy M., Aoki W., et al. 2004 A\& A 417, 293

Arkhipova V.P., Ikonnikova N.P., \& Noskova R.I. 1999 Ast. Lett. 25, 25

Arkhipova V.P., Klochkova V.G., \& Sokol G.V. 2001a Ast. Lett. 27, 99

Arkhipova V.P., Ikonnikova N.P., Noskova R.I., et al. 2001b Ast. Lett. 27, 719

Bianchi L., et al. 2001 AJ 122, 1538

Blöcker T. 1995 A\& A 299, 755

Bobrowsky M., Sahu K.C., Parthasarathy M., \& Garcia-Lario P. 1998 Nature 392, 469

Bond H.E. 1991 IAU Symposium 145, 341

Borkowski K.J., \& Harrington J.P. 2001 ApJ 550, 778

Branch D., Livio M., Yungelson L.R., et al. 1995 PASP 107, 1019

Cami J., \& Yamamura I. 2001 A\&A 367, L1

Cohen M., Van Winckel H., Bond H.E., et al. 2004 AJ 127, 2362

de Boer K.S. 1987 IAU Colloq. 95, 95

De Ruyter S., et al. 2006 A\&BA 448, 641

Deroo P., et al. 2005 A $\& A 438,987$

Fiebelman W.A 1995 ApJ 443, 245

Gauba G., \& Parthasarathy M. 2003 A $\& A$ 407, 1007

Gauba G., \& Parthasarathy M. 2004 A\&S 417, 201

Garcia-Lario P., Parthasarathy M., De Martino D., et al. 1997 A $\& A$ 326, 1103

Giridhar S., Lambert D.L., Reddy B.E., et al. 2005 ApJ 627, 432

Gonzalez G., \& Wallerstein G. 1992 MNRAS 254, 343

Hamuy M., et al. 2003 Nature 424, 651

Harris H.C., Nemec J.M., \& Hesser J. 1983 PASP 95, 256

Jasniewicz G., de Laverny P., Parthasarathy M., et al. 2004 A $\mathscr{G} A$ 423, 353

Jura M. 2005 ASP Conf.Ser. 337, 191

Jura M., Velusamy T., \& Werner M.W. 2001 ApJ 556, 408

Klochkova V.G., et al. 2002 Ast. Lett. 28, 51

Klochkova V.G., Yushkin M.V., Miroshnichenko A.S., et al. 2002 A $\mathscr{J} A$ 392, 143

Kotak R., et al. 2004 MNRAS 354, L13

Kwok S. 1993 ARA $\& A$ 31, 63

Maas T., et al. 2003 A\&A 405, 271

Maas T., Van Winckel H., \& Evans T.L. 2005 A $\& A$ 297, 308 
Moehler S., Heber M., Lemke M., et al. 1998 A $\& A$ 339, 537

Molster F.J., et al. 1999 A $\mathscr{G} A$ 350, 163

Monier R., \& Parthasarathy M. 1999 A\& A 341, 117

Mooney C.J., Rolleston W.R.J., Keenan F.P., et al. 2002 MNRAS 337, 851

Mooney C.J., Rolleston W.R.J., Keenan F.P., et al. 2004 A $\mathscr{E} A$ 419, 1123

Parthasarathy M. 1993a ApJ 414, L109

Parthasarathy M. 1993b ASP Conf.Ser. 45, 173

Parthasarathy M. 1994 ASP Conf.Ser. 60, 261

Parthasarathy M. 1999 IAU Symp. 191, 475

Parthasarathy M., \& Pottasch S.R. 1986 A $\& A$ 154, L16

Parthasarathy M., \& Pottasch S.R. 1989 A $\mathscr{E} A$ 225, 521

Parthasarathy M., Garcia-Lario P., \& Pottasch S.R. 1992 A $\& A$ 264, 159

Parthasarathy M., Garcia-Lario P., Pottasch S.R., et al. 1993 A $6 A$ 267, L19

Parthasarathy M., Garcia-Lario P., de Martino D., et al. 1995 A $\& A$ 300, L25

Parthasarathy M., Vijapurkar J., \& Drilling J.S. 2000a $A \mathscr{E} A S$ 145, 269

Parthasarathy M., Garcia-Lario P., Sivarani T., et al. 2000b A\&A 357, 241

Parthasarathy M., Garcia-Lario P., Gauba G., et al. 2001a A\& $A$ 376, 941

Parthasarathy M., Gauba G., Fujii T., \& Nakada Y. 2001b in Post-AGB objects as a phase of stellar evolution, 29, (eds Szczerba \& Gorny :Kluwer)

Pereira C.B. 2004 A $\xi A$ 413, 1009

Pereira C.B., et al. 2004 A $\mathscr{E} A$ 422, 637

Pottasch S.R., \& Parthasarathy M. 1988 A\&A 192, 182

Reddy B.E., \& Hrivnak B.J. 1999 AJ 117, 1834

Reddy B.E., Parthasarathy M., Gonzalez G., \& Bakker E.J. 1997 A $\& A$ 328, 331

Reddy B.E., Lambert D.L., Gonzalez G., \& Yong D. 2002 ApJ 564, 482

Reyniers M., et al. 2002 A\&SA 395, L35

Reyniers M., \& Van Winckel H. 2001 A $\& A$ 365, 465

Reyniers M., Van Winckel H., Gallino R., \& Straniero O. 2004 A\&SA 417, 269

Riera A., et al. 2003 A\&A 401, 1039

Ryans R.S.I., Dufton P.L., Mooney C.J., et al. 2003 A\&A 401, 1119

Sahai R., Bujarrabal V., \& Zijlstra A. 1999 ApJ 518, L115

Sarkar G., Parthasarathy M., \& Reddy B.E. 2005 A\&A 431,1007

Schönberner D. 1983 ApJ 272, 708

Schultheis M., Parthasarathy M., Omont A., et al. 2002, A $\mathscr{E} A$ 386, 899

Sivarani T. 2000 Ph.D thesis Bangalore University

Sivarani T., Parthasarathy M., Garcia-Lario P., et al. 1999 A $\& A S$ 137, 505

Takeda Y., Parthasarathy M., Aoki W., et al. 2002 PASJ 54, 765

Thompson H.M.A., Keenan F.P., Dufton P.L., et al. 2006 MNRAS 368, 1749

Thevenin F., Parthasarathy M., \& Jasniewicz G. 2000 A $\mathscr{E} A$ 359, 138

Turner D.G., \& Drilling J.S. 1984 PASP 96, 292

Ueta T., Meixner M., \& Bobrowsky M. 2000 ApJ 528, 861

Van Winckel H. 2003, ARA\&A 41, 391

Van Winckel H., Cohen M., \& Gull T.R. 2002 AESA 390, 147

Van Winckel H., Mathis J.S., \& Waelkens C. 1992, Nature 356, 500

Volk K.M., \& Kwok S. 1989, ApJ 342, 345

Waelkens C., Van Winckel H., \& Waters L.B.F.M., et al. 1996, AEGA 314, L17

Waelkens C., Waters L.B.F.M., \& Van Winckel H. 1995, ApSS 224, 357

Wang L., et al. 2004, ApJ 604, L53

Witt A.N., \& Vijh U.P. 2004, ASP Conf.Ser. 309

Wood-Vasey W.M., et al. 2004, ApJ 616, 339

Zinn R.J., Newell E.B., \& Gibson J.B. 1972, A\&A 18, 390 